XV.

\title{
Ueber Tabes dorsalis beim weiblichen Geschlecht.
}

Yon

\author{
Dr. H. Kron, \\ Nervenarzt in Berlin.
}

Dass weibliche Tabesfälle dem Nachweise früherer syphilitischer Infection oft die grössten Schwierigkeiten entgegensetzen, ist bekannt. Dafür bietet aber die einfachere Lebensweise der Frauen, das Fortfallen gewisser Schädlichkeiten, wie Alkohol, Tabak, Excesse etc., einen weit klareren Ueberblick über die möglichen anderweitigen Krankheitsursachen. Deshalb sollte man von der Verwierthung dieses Materials nicht abstehen. Freilich darf man, was auch Erb und Möbius betont haben, die weiblichen Fülle nicht mit den männlichen vermengen.

Wir finden in der Literatur nur eine kleine Anzahl von Arbeiten, die sich in dieser Weise mit weiblichen Tabikern beschäftigen. Sehen wir von 11 Fällen, die Leonhard ${ }^{1}$ ) vor der berübmten Fournierschen Abhandlung aus Westphal's Klinik zusammengestellt, und der wohl von keiner Seite für massgebend erachteten Statistik Lothar Meyer's ${ }^{2}$ ) ab, so lieferte $\mathrm{Erb}^{3}$ ) den ersten Beitrag: Unter 13 tabischen Frauen 4 mit sicherer, 5 mit höchstwahrscheinlicher Syphilis, eine mit einem Schanker, 3 zweifelhafte. Mit Fortlassung dieser, also unter 10 Fällen, 4 ohne, 6 mit Infection. Diesen Angaben fügte dann Mö biu s${ }^{4}$ ) 18 Fälle hinzu, in deren Anamnese sich fast durchweg Syphilis nachweisen liess [5 mit 80 Proc., 13 mit 93 Proc.], während. andere causale Momente häufig ganz feblten. Mendel's poliklinisches Ma-

1) Berliner Dissertation 1872.

2) Archiv f. Psychiatrie und Nervenkrankheiten. 1880. S. 252.

3) Berliner klinische Wochenschrift. 1883. S. 483.

4) Centralblatt für Nervenheilkunde. 1894. Nr. 9, 12, 20 und Neurologische Beiträge. Heft 3, S. 108. 
terial fand einige Jahre später in Hlubek ${ }^{1}$ ) seinen Bearbeiter, der unter den 30 weiblichen Tabikern bei 46 Proc. voraufgegangene Syphilis fand, was Minor ${ }^{2}$ ) in allen seinen 8 Fällen, wenn man einen kaum zweifelhaften mit hinzurechnet, gelang. Bald trat auch $\mathrm{Erb}^{3}$ ) wieder mit Beobachtangen hervor: Unter den 19 weiblichen Tabesfällen hatten 9 sicher, 8 fast sicher Syphilis gehabt. Weiter waren unter 21 neuen Fällen von Möbius $\left.{ }^{4}\right) 18$ mit sicherer oder doch höchstwahrscheinlicher Syphilis (86 Proc.). 54,4 Proc. rechnete Friedrichsen ${ }^{5}$ ) aus 33 Fällen der Gerhardt'schen Klinik heraus. In einer letzten Reihe von 7 Fällen hatte Möbius ${ }^{6}$ ) 100 Proc. früherer Syphilis, während Erb ${ }^{7}$ ) unter seinen neuesten 9 Fällen 6 mit ganz sicherer, 2 mit ziemlich sicherer und 1 mit höchst wahrscheinlicher Infection nachwies. Von den Autoren, die bei ihren, männliche und weibliche Fälle umfassenden, Arbeiten der letzteren besonders gedenken, will ich hier nur Gerlach ${ }^{8}$, Bernhardt ${ }^{9}$ ), Tumpowsky ${ }^{10}$ ), Redlich ${ }^{11}$ ) anführen.

Ich lasse nunmehr meine eigenen Beobachtungen in aller Kürze folgen:

1. Elise R. 46 jāhrige Arbeiterin. Sy mptome: refl Pupillenstarre, Westphal'sches Zeichen, Hypalgesie an den unteren Extremitäten, Blasenparese, Complication mit Bulbärparalyse (später hinzugetreten). - Am Unterschenkel grosse glatte Narbe, voll einem Ulcus, das erst unter Quecksilbersalbe geheilt ist.

2. Therese B. 40 jahrige Kaufmannsfrall. 5 Geburten, 1 Abort. Letzte Entbindung im 39. Jahre, Kind viel rothe Flecke auf der Haut, lebte nur 6 Stunden. - Im 39. Jahre Schwäche des linken Beines. Symptome: Pupillen ungleich, lichtstarr, Westphal'sches Zeichen. Analgesie an den Unterschenkeln, Blasenparese, Migriane, Schwindel.

3. Emilie Fr. 39jahrige Sclneiderin, Wittwe. (Nühmaschine nur im ersten Jahre der Ehe.) Mit 18 Jahren geheirathet. In Beginn der Ehe sofort „Gebårmutterentzündung". Danach 4-5 Aborte, dann Sterilität. (Ehemann im 26. Jahre der Pat. geschlechtskrank, bald daranf heiser, starb an einer „Nervenkrankheit's.) Mit 36 Jahren ausgebreitetes Exanthem. Im 38. Jahre lanzinirende Schmerzen, Sehstörung. Intervall 20 Jahre. Symptome: Pupillen ungleich, lichtstarr, Kniephänomen fehlt rechts, ist links

1) Berliner Dissertation. 1886.

2) Contribution à l'étude de l'étiologie du tabes. Arch. de neurologie. 1859. S. 391.

3) Berliner klinische Wochenschrift. 1891.

4) Centralblatt für Nervenheilkunde. Sept. 1893 und Neurologische Beiträge.

Heft 3. S 132 .

5) Berliner Dissertation. 1893.

6) Neurologische Beiträge. Heft 3. S. 138.

7) Berliner klinische Wochenschrift. 1596. Nr. 11.

8) Syphilis and Tabes. Hallenser Dissertation. 1890.

9) Centralblatt für Nervenheilkunde. 1883. S. 457.

10) Deutsche Zeitschrift für Nervenheilkunde. Band 10. Heft $5 / 6$.

11) Die Pathologie der tabischen Hinterstrangserkrankung. Jena 1897. 
nur mit Jendrassik zu erzielen. Hypästhesie an den unteren, Hypalgesie an den oberen Extremitäten.

4. Luise H., 35jährige Schneiderin. Mit 30 Jahren verheirathet, steril; im 21. Jalure Syphilis, Spritzcur in der Charité. Mit $30 \mathrm{~J}$ ahren lanzinirende Schmerzen in den Beinen. Intervall 9 Jahre. Symptome: Erhebliche Ungleichheit der Papillen, minimale Reaction auf Licht. Westphal'sches Zeichen, Analgesie an den Unterschenkeln.

5. Dorothea R., 54jährige Kaufmannsfrau. 2 Kinder früh gestorben, 3 Aborte. Mit 51 Jahren Migräne. Sy mp tome: Pupillen sehr eng, lichtstarr. IVestphal'sches Zeichen. Anästhesie an den nnteren Extremitäten. Blasenparese.

6. Marie P., 39jährige Kanfmannsfrau. Steril. Symptome: Zuerst lanzinirende Schmerzen in den Beinen. - Pupillen lichtstarr, Westphalsches Zeichen. Leichte Ataxie, Blasenparese, Schwindel.

7. Elise U., 34jührige Schneiderin (nur mit Handmaschine beschäftigt); verheirathet seit 20 . Jahre, 4 Geburten, darunter 3 Aborte. Mann im 24. Jahre der Pat. geschlechtskkrank gewesen. Coitus nicht eingestellt. Im 25. Jahre rothe Flecke auf der Haut, die mit Jodkalium behandelt wurden. Während des Exanthems Frühgeburt von 7 Monaten. Im 30. Jahre lanzinirende Schmerzen in den Beinen und Schultern, Kopfschmerz, Schwindel. Intervall 6 Jahre. Symptome: Papillen eng, lichtstarr, Westphal'sches Zeichen. Analgesie an den Unterschenkeln, Ro'mberg's Symptom, Blasenparese, Larynxkrisen.

8. Johanna A., 38jährige Schneiderin, verheirathet mit 20 Jahren; steril. Mit 22 Jahren Infection (Ulcera an den Labien). Subcut. Einspritzungen, Pillen. Scheidung deswegen nach 2 jähriger Ehe. Im 32. Jah re Schlaff heit der Beine, viel Schweiss, wenig Schlaf. Intervall 10 Jahre. Symptome: Pupillen ungleich, sehr eng, starr. Westphal'sches Zeichen. Hypalgesie an den Unterschenkeln.

9. Karoline B., 54jährige Arbeiterin. Seit Ende der 20. Jahre verheirathet. Wittwe seit dem 49. Jahre. 2 Geburten, ein Kind gestorben. Im 53. Jahre Sturz 11/2 Etagen tief aus einem Fenster. Im 54. Jahre lanzinirende Schmerzen in den Beinen. Symptome: Pupillen lichtstarr, Westphal'sches Zeichen, lanzinirende Schmerzen in den Beinen. Sensorischsensible Hemianaesthesia dextra. Blasenparese, Parese des rechten Beines.

10. Auguste L., 38jahbrige Schneiderin (Nähmaschine vom 24.-30.Jahre, später nicht mehr). Mit 33 Jahren verheirathet, steril. Mann vor und in der Ehe geschlechtskrank gewesen. Nach 3jähriger Ehe deswegen Scheidung. Infection wahrscheinlich im Beginne der Ehe. Mit $36 \mathrm{Jahren}$ Ptosis, Strabismus divergens. Intervall 2-3 Jahre. Symptome: Pupillen lichtstarr, Westphal'sches Zeichen links, lanzinirende Schmerzen, Analgesie an den Beinen, Strab. divergens, doppelseitig, besonders rechts.

11. Johanna $G_{\text {., }}$ 48jährige Plätterin, ledig. (Mutter geisteskrank gewesen.) Mit 25 Jahren Infection (Condyl. lata). Mit $38 \mathrm{Jahren} \mathrm{Schmerzen}$ in Stirn, Nacken und Beinen. Intervall 13 Jahre. Symptome: Westphal' sches Zeichen, lanzinirende Schmerzen in den Beinen, Analgesie daselbst, Gürtelschınerz, Kopfschmerz, Schwindel, Angstgefühl. Polydipsie, Blasenparese.

12. Marie Z, 30jährige Schneiderin (nie Maschine genäht), ledig. Vater an Paralyse gestorben. Mit 21 Jahren Infection (erst nach Jahren einge- 
standen). Damals nur ausserlich behandelt, später, schon nach Beginn der Tabes, mehrere kurze Schmiercuren. Symptome: Pupillen ungleich, linke reagirt etwas träge. Westphal'sches Zeichen. Blasenparese, hochgradige Ataxie der unteren, leichte der oberen Fxtremitäten, Anästhesie an den unteren.

13. Charlotte Fl., 55jâhrige Schlăchtersfrau. Seit dem 20. Jahre verbeirathet, steril, giebt als Ursache der Tabes Erlältungen an. Mit 45 Jahren Doppeltsehen, mit 46 beginnende Opticusatrophie. Symptome: Papillen lichtstarr, Opticusatrophie, Westph al'sches Zeichen. Parästhesien an den Füssen. Complication mit Diabetes mellitus (anhaltend bis 4,3 Proc.).

14. Flora W., 58jăhrige Kanfmannsfran, verheirathet seit dem 27. Jahre. 8 lebende Kinder, 2 Aborte. Im 48. Jahr Ulcns durum an der Lippe durch Kuss. Injectionscur. Mit $52 \mathrm{~J}$ ahren lanzinirende Schmerzen im Nacken and Hinterkopf. Intervall: 4 Jahre. Symptome: Pupillen lichtstarr, Kniephänomen vorhanden (Tabes super.), leichte Ataxie der oberen Extremitäten, lanzinirende Schmerzen in denselben and an den angegebenen Stellen.

15. Marie Z-r., 50jāhrige Schneiderin, verheirathet. Mann an Paralyse gestorben, hatte früher, im 33. Jahre der Pat., Syphilis. 17 Geburten, darunter 6 Frühgeburten and verschiedene Aborte. Viel Nähmaschine, Gemüthsbewegungen, Anstrengung durch Unterricht. 4 Kinder leben. Wahrscheinlich Infection in 33. Jahre. Mit $44 \mathrm{Jahren}$ lanzinirende Schmerzen in den Beinen. In terva1l: 11 Jahre. Jodkalium im 47. Jahr. Sy mptome: Pupillen eng, starr, Westphal'sches Zeichen. Analgesie an den unteren Extremitäten Gürtelschmerz, Blasenparese.

16. Anna Bl., 36jăhrige Schneiderin, ledig, vom 21.-24. Jahre sexueller Verkehr. Mit 24. und 30. Jahre Drüsenanschwellungen oberhalb der Schlüsselbeine. Nie Maschinenarbeit. Mit 28 Jahren Schmerzen im Rücken und in den Beinen. Symptome: Pupillen lichtstarr, Westphal'sches Zeichen, lanzinirende Schmerzen in den Beinen, Blasenparese. Hïnfig Plaques anf der Zunge.

17. Lnise S., 39jährige Schneiderin, ledig. Vom 35.-36. Jahre sexueller Verkehr. Brennen beim Urinlassen zn jener Zeit. - Neuropathisch belastet (Vater nervenleidend gewesen). Angestrengtes Maschinenähen vom 16. Jahr an. Mit 38 Jahren lanzinirende Schmerzen in den Beinen, Intervall event. 3 Jahre. Symptome: Pupillen starr, nngleich, links Westpha l'sches Zeichen, lanzinirende Schmerzen, Blasenparese. Complication mit Morbas Basedowii.

18. Anguste $\mathrm{Kl}$., 58jährige Schneiderin. Seit dem 23. Jahre verheirathet. 2 Kinder leben, 3 mit 7 Monaten abgestorben. 25 Jahre angestrengtes Maschinenăhen. starke einmalige Durchnässung nnd Erkïltung. Im 40. Jahre Flecke auf der Hant. Mit 43. Jahr einseitige Mydriasis. Sy m p to m e: Pupillen erheblich ungleich, lichtstarr, Westphal'sches Zeichen, Romberg's Symptom, lanzinirende Schınerzen in Beinen nnd rechtem Arm mit Gefiuhl von Taubsein daselbst, Andsthesie an der linken Unterextremitä, Polydipsie. Anf Schnltern and Hals syphilitisches Exanthem (Dr. A. Weyl).

19. Ida B., 28jahrige Schneiderin, mit 18 Jahren verheirathet, mit 23 Jahren nach acut aufgetretenem, sehr starkem Ausfluss Abort, mit 25 Jahren geschieden wegen roher Behandlung seitens des Mannes. 2 Kinder, 1 Abort. Vater an Nervenleiden gestorben. Angestrengtes Maschinenähen seit 4 Jahren. Seit dem 25. Jahro Anfalle von Bewusstlosigkeit. Symptome: Pupillen weit, lichtstarr, Kniephänomen links schwach, Blasenparese, Opticusatrophie.

20. Panline B, 46jăhrige Schneiderin, ledig; mit 23 Jahren zum ersten, mit 25 Jahren zam zweiten Mal uneheliche Geburt. Ueberarbeitet durch 
Sticken, keine Maschinenarbeit. Mit 41 Jahren tanbes Gefühl in den Zehen. Symptome: Pupillen eng, starr, Westphal'sches Zeichen, erhebliche Ataxie, Analgesie an den Unterschenkeln, Blasenparese.

21. Franziska B., 32jährige Schneiderin, ledig. Mit 29 Jahren Roseola und Angina specifica. 3 Wochen Schmiercur, danach ein Vierteljahr-Jodkalinm. Maschine nur hin und wieder, sehr wenig. Bald nach dem Exanthem lanzinirende Schmerzen in den Beinen. Symptome: Pupillen ungleich, lichtstarr, Kniephänomen schwach, besonders links. Analgesie an den unteren Extremitäten, Gürtelgefühl, Romberg, Mastdarmschmerz. Plaques an der Zunge, vordere und hintere Cervicaldrüsen geschwollen (Dr. A. Blaschko). 22. Bernhardine J., 46jührige Handelsfrau. Seit dem 19. Jahre verheirathet. 10 Geburten, 3 in den ersten Monaten gestorben, 2 Aborte, körperliche Anstrengungen, Erkältungen. Mit 34 Jahren Taubheitsgefïhl im Bereiche des rechten N. cut. fem. ext. Parästhesie im linken Ulnarisgebiet. Sy mp to me: Pupillen starr, sehr eng, W es tphal'sches Zeichen, starke Ataxie, Aufhebung des Muskelgefühls an den unteren Extremitäten, Opticusatrophie, Blasenparese, Mastdarmschınerz.

23. Gottliebe Chr., 45jährige Kautmannsfrau. Terheirathet zum 1. Mal mit 26 Jahren. 1 gesundes Kind, dann mehrere Jahre Pause, darauf 4 Kinder, die alle unter einem Jahre, bezw. wenige Stunden nach der Geburt starben. Zum 2. Mal verheirathet mit 40 Jalıren, 2 gesunde Kinder. Erster Mann an Paralyse gestorben. Infection wahrscheinlich im Beginne der ersten Ehe. Mit 44 Jahren lanzinirende Schmerzen in den Beinen. Intervall: 18 Jahre. Symptome: Pupillen lichtstarr, ungleich, Westphal'sches Zeichen, Parästhesien an den Füssen, Romberg, Blasenparese. 24. Luise Sch., 49jährige Waschfran. Verheirathet seit dem 19. Jahr, 3 gesunde Kinder, Mann im 29. Jahre der Pat. geschlechtskrank, danach keine Geburt mehr, Erkältungen. Mit 46 Jahren Migräne, lanzinirende Schmerzen, gastrische Krisen. Intervall eventuell 17 Jahre. Symptome: Papillen lichtstarr, Kniephänomen schwach, Analgesie, lanzinirende Schmerzen an- den unteren Extremitiaten, Blasenparese.

25. Elise $\mathrm{Kr}$., 42 jäbrige Schneiderin, verheirathet mit 18 Jahren. 8 Kinder, 4 unter einem Jahr gestorben. Viel Maschinenähen. Im 38. Jahre Schwindel, Kopfschmerz. Symptome: Einseitige Pupillenstarre, Westphal'sches Zeichen, lanzinirende Schmerzen, Blasenparese.

26. Dorothea Z., Hebamme, 55jälrige Wittwe. 21jährigen Sohn. Mit 45 Jahren Infection am Finger bei Ausübung des Berufs, danach Lymphadenitis in der Achsel, zwei Monate später Hauteruption, ein halbes Jahr danach Plaques auf der Zunge. Injectionscur im Beginne der Lues, Schmiercur beim Recidiv. Mit $50 \mathrm{~J}$ ah ren lanzinirende Schmerzen im rechten Arm. Intervall 5 Jahre. Symptome: Pupillen eng, ungleich, starr, West phal'sches Zeichen, Ataxie, Analgesie, Gürtelschmerz, Abducensparese rechts, Ptosis links.

27. Karoline N., 51 juhrige Schneiderin, verheirathet seit dem 19. Jahre; geschieden im 29. Jahre wegen Geschlechtskrankheit des Mannes. 1 Kind mit 11 Monaten an Krämpfen gestorben, 3 Aborte. Nach der Verbeirathung längere Zeit „Blasen" anf den Lippen. Nit $46 \mathrm{~J}$ ah ren lanzinirende Schmerzen, Gürtelgefïhl. Sy mpt om e: Pupillen sebr eng, starr, Wes tphal'sches Zeichen, lanzinirende Schmerzen in Armen und Beinen, leichte Ataxie der ersteren, Gürtelgefühl, Parästhesien in den Fingern. 
28. Elise Sch., 32jahrige Schneiderin. Verheirathet mit 24 Jahren. Steril. Mit 18 Jahren eitrige Affection an den änsseren Genitalien. Nenropathisch belastet, nie Maschine genäht. Mit 27 Jahren lanzinirende Schmerzen in den Beinen, Intervall 9 Jahre. Symptome: Pupillen lichtstarr, ungleich, W es tph al'sches Zeichen, Analgesie, Ataxie der unteren Extremitäten, Blasenparese, Ptosis rechts.

29. Lina Schm., 50jahrige Schneiderin, verheirathet mit 24 Jahren, steril. Seit 11 Jahren angestrengtes Maschinenähen. Mit 40 Jahren Krämpfe in den Füssen, dann Rückenschmerzen, gastrische Krisen. Symptome: Pupillen lichtstarr, Westphal'sches Zeichen, Romberg, Analgesie der unteren Extremitäten, Blasenparese.

30. Bertha Sch., 40jahrige Schneiderin. Verheirathet mit 29 Jahren. 1 Kind von 8 Jahren. 2 Jahre angestrengtes Maschinenähen, nur 1 Jahr vor Ausbruch der Krankheit. Mit 38 Jahren Kopfschmerz, Schwindel. Symptome: Pupillen ungleich, starr, Westphal'sches Zeichen, Analgesie an den Unterschenkeln.

31. Anguste St., 46jahrige Kaufmannsfrau, verheirathet mit 28 Jahren, steril. Mann syphilitisch gewesen, jetzt ebenfalls Tabiker. - Mit 29 Jahren Roseola specif.; Injectionscur. Mit 32 Jahren Schwäche des linken Beines. Intervall 3 Jahre. Symptome: Pupillen weit, ungleich, lichtstarr; Westphal'sches Zeichen, lanzinirende Schmerzen, Analgesie der unteren Extremitaten, Romberg, gastrische Krisen, Blasenparese.

32. Elisabeth Sch., 36jahrige Kanfmannsfrau. Mann syphilitisch gewesen. 10 Geburten, 5 Kinder im frühesten Lebensalter an Krämpfen gestorben, 1 mit Ansschlag geboren, der im 15. Jahre recidivirte, ein anderes Kind ebenfalls mit Ausschlag geboren, im 2. Jahre fast erblindet, ärztlicherseits für syphilitisch erklärt. - Mit 32 Jahren Gürtelschmerz, Brechneigung. Sy mp t o me: Pupillen ungleich, starr, Kniephänomen schwach, hăufige schwere gastrische Krisen, Gürtelgefühl, lanzinirende Schmerzen im Nacken and Rücken.

33. Anna N., 39jährige Näherin, ledig. Im 16. Jahre sexueller Verkehr, 1 Kind, nach 4 Monaten gestorben. (Die 2 Kinder, die der betreffende Mann in seiner späteren Ehe mit einer anderen gezengt, sind nach $1 \frac{1}{2}-2$ Jahren gestorben.) In den letaten 7 Jahren anstrengendes Maschinenähen. Mit 37 Jahren lanzinirende Schmerzen. Sy mp to me: Pupillen eng, ungleich, starr, Westphal'sches Zeichen, Analgesie an den unteren Extremitäten.

34. Lnise G., 42jăhrige Portiersfrau. Verheirathet mit 29 Jahren, 2 Kinder leben, 1 nach 8 Wochen, 1 nach 3 Tagen gestorbeu, 1 Abort. Der Mann giebt zu, im Beginne der Ehe eine leichte Gon. gehabt zu haben. - Mit 31 Jahren Halsaffection, mit 32 Jahren Recidiv mit Kehlkopfsbetheiligung (Tracheotomie); im folgenden Jahre Periostitis septi narium, Ulcerationen an Zunge and Epiglottis (Angaben des Herrn Dr. Saatz). Jodkalium zar Zeit der Infection. Mit $33 \mathrm{~J}$ ahren lanzinirende Schmerzen in den Beinen. Intervall 4 Jahre. Sy mptome: Pupillen eng, ungleich, lichtstarr, Wostphal'sches Zeichen, Analgesio der unteren Extremităten, Blasenparese. 35. Marie Bl., 40jahrige Schneiderin, verheirathet mit 31 Jahren. Mann in der Ehe geschlechtskrank geworden, 2 Kinder, im 5. und 6. Monat gestorben, 1 Abort. Mit 33 Jahren lanzinirende Schmerzen in den Beinen. Symptome: Pupillen eng, starr, Westphal'sches Zeichen, Analgesie an den Unterschenkeln, Parästhesio an der linken Hand, Blasenparese. 
36. Luise Sch., 49jährige Schneiderin. Verheirathet mit 22 Jahren, 1 Kind mit $1 / 4 \mathrm{Jahr}$ an Krämpfen gestorben, dann 1 Abort, von da an steril. (Mann lüderlich, Umgang mit anderen Frauen.) 27 Jahre lang schweres Maschinenähen, mit $42 \mathrm{~J}$ ahr en Schmerz im Rücken, Schwindel, Erbrechen. Symptome: Pupillen ungleich, lichtstarr, Kniephänomen sehr schwach, Gürtelschmerz, Blasenparese.

37. Marie $\mathrm{Kr}$., 42 jährige Schneiderin, mit 18 Jahren verheirathet. 1 nneheliches Find von 2 Jahren gestorben, seitdem steril. Ca. 15 Jahre angestrengtes Naschinenähen, mit $27 \mathrm{~J}$ ahren lanzinirende Schmerzen in den Beinen. Sy mp tom e: Papillen eng, starr, Kniephänomen links schwach, Analgesie an den unteren Extremitäten, heftiger Kopfschmerz.

38. Minna L., 32jührige Schneiderin. Verheirathet mit 29 Jahren, steril, nie Maschine genïht; mit 30 Jahren heftiger Kopfschmerz. Symptome: Pupillen erheblich ungleich, starr, Kniephïnomen vorhanden, lanzinirende Schmerzen, Analgesie der Beine, Gürtelschmerz, Kopfschmerz.

39. Anna K., 38jährige Kaufinannstran. Verheirathet seit 29. Jahre, steril, Infection im 23. Jahre, im 27. Jahre Jodkaliumcur. Neuropathisch belastet (Vater Potator, Bruder Paralytiker). Mit 26 Jahren Doppeltsehen. Intervall 3 Jahre. Symptome: Pupillen ungleich, starr, Opticusatrophie mit Amaurose, Westphal'sches Zeichen, Analgesie an den unteren Extremitäten, Romberg. - In der linken Inguinalgegend strahlige Narbe. Mann, der zugiebt, in den 20 er Jahren Gon. gehabt und damals mit Pat. verkehrt zu haben, jetzt ebenfalls Tabiker.

40. Henriette M., 34jahrige Schneiderin. Verheirathet mit 17 Jahren, verwittwet mit 22 Jahren, sexueller Verkehr tortgesetzt. 3 Kinder. Angestrengtes, lang fortgesetztes Maschinenähen. Mit 32 Jahren heftiger Kopfschmerz, Schwäche. Symptome: Pupillen ungleich, lichtstarr, Westphal'sches Zeichen, Gürtelschmerz, Romberg, Blasenparese - Verdachtsmomente für Syphilis (Dr. O. Rosenthal): Starke Kopfschmerzen, Alopecie, Schwellnng der Cervical-, Occipital- und Inguinaldrüsen.

41. Anna K., 32 jïhrige Arbeiterin, verheirathet seit 19. Jahre. 3 Kinder, 2 leben, 2 Aborte. Infection im 18. Jahre. Keine Maschinenarbeit. Im 29. Jahre einseitige Ptosis. Bei den Infectionserscheinungen Theecur. Intervall 11 Jahre. Symptome: Pupillen ungleich, eine starr, die andere träge, Westphal'sches Zeichen, Analgesie der unteren Extremitäten, Ataxie, Blasenpareso.

Die Betrachtung dieser 41 Fälle ergiebt nun Folgendes:

1. Beschäftigung. Wir finden darunter 24 Schneiderinnen, 8 Kaufmannsfrauen, 1 Hebamme, 1 Waschfrau, 1 Portiersfran, 1 Wäscheplätterin, 1 Schlächtersfrau, 3 „Arbeiterinnen“, 1 Handelsfrau. Das Material ist zum überwiegenden Theile der hiesigen Ortskrankenkasse der Schneider entnommen. Daher die grosse Zahl der Kranken aus diesem Berufszweige.

2. Lebensalter im Beginne der Krankheit. In 38 Fällen liess sich dasselbe mit der Sicherheit ermitteln, die man hierbei erwarten kann. Dannach fallen in das Alter von 25-30 Jahren 11 Fälle, 31-40: 16, 41-50: 9, 51-54: 3 Fälle. Die jüngste Patientin war 25, 
die älteste 54 Jahre, als sich die ersten Erscheinungen der Tabes bemerklich machten. Die meisten Fälle liegen also auch hier zwischen dem 30. und 40. Jahre, entsprechend den Befunden von Erb, Eulenburg, Möbius u. A. [nach letzterem1) ca. 2/3]. Wir haben aber auch verhältnissmässig nicht wenig (10 Fälle) zwischen dem 25 . und 30 . Jahre zu verzeichnen.

3. Civilstand. Es waren verheirathet 33, ledig 8. Von letzteren hatten 7 sexuellen Verkehr gehabt, für die übrigbleibende ( $\mathrm{Nr} .1$ ) ist dies in hohem Grade wahrscheinlich.

4. Geburtsverhältnisse. Bei den 33 Verheiratheten bestand Sterilität in 10 Fällen $=30,3$ Proc. Fällt hier schon die grosse Zahl der sterilen Ehen auf, so nicht minder auch die Häufigkeit der Aborte, selbst wenn man die Gepflogenheiten der betreffenden Kreise nach dieser Richtung hin in Rechnung zieht, nämlich 37 gegen 84 rechtzeitige Geburten, ganz besonders aber die Sterblichkeit der Kinder unter einem Jahre, 22 von 27 Todesfällen $=81,5$ Proc. Die Erklärung dafür finden wir in der

5. Aetiologie. Ich benutze hier das bekannte Schema Erb's. Danach fand sich

Syphilis allein . . . . . . . . . . $14 \mathrm{mal}=34$ Proc.

(Fall 3. 4. 7. 8. 11. 14. 21. 26. 27. 31. 32. 34. 39. 41)

Syphilis and neuropathische Disposition . . . 2 mal (Fall 12 u. 28)

Syphilis und körperliche Anstrengung und Er-

kältung . . . . . . . . . . . $1 \mathrm{mal}$ (Fall 1S)

Syphilis und Erkältung . . . . . . . . 1 mal (Fall 24)

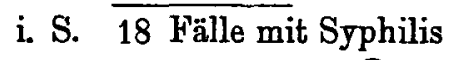
$=44$ Proc.

Dazu kommen noch 3 Fälle (10. 23. 35) mit wahrscheinlicher Syphilis allein und 2 (15 und 17) mit wahrscheinlicher Syphilis und körperlicher Anstrengung. Rechnen wir diese Fälle, in denen doch eigentlich kaum ein Zweifel obwaltet, noch hinzu, so erhalten wir 23 Fälle $=56$ Proc. mit Syphilis in der Vorgeschichte.

Weiterhin sind 7 Fälle verzeichnet, in denen keine Syphilis nachzuweisen war, aber auch keine andere Ursache. Der Umstand, dass alle 7 Verdachtsmomente, zum Theil erheblicher Art, bieten, lässt sie keineswegs zweifellos erscheinen. Es sind dies Fall 1. 2. 5. 6. 16. 20. 38. Ferner liegen 5 Fälle mit anderen Ursachen, aber auch gewissen Verdachtsmomenten vor: Fall 19. 33. 36. 37. 40.

Ich rechne diese 12 Fälle nicht hinzu, möchte aber doch nicht anterlassen, auf sie hinzuweisen.

1) Ueber die Tabes. Berlin 1897. 
An ätiologischen Momenten ausser der Syphilis finden wir:

Körperliche Anstrengungen . . . . . . . . . . $7 \mathrm{mal}$ (Fall 20.

25. 29. 33. 36. 37. 40$)$

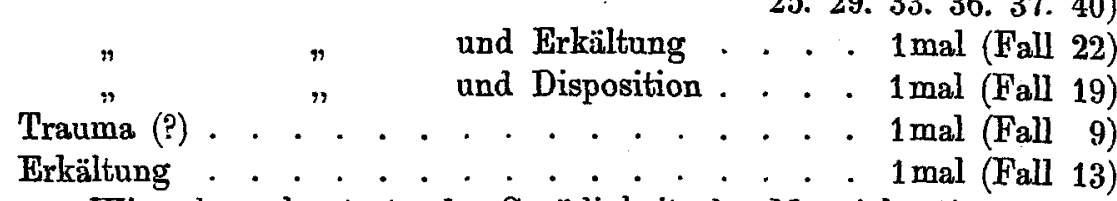

Wir sehen also trotz der Sprödigkeit des Materials die anderen möglichen Ursachen vor der Syphilis ganz erheblich in den Hintergrund treten.

In dem Fall 9 hat vor dem Unfall bereits Blasenstörung bestanden, er bestätigt also nur die von $\mathrm{Mendel}^{1}$ ) erhärtete Thatsache, dass Trauma nicht zur Tabes führt.

Neuropathische Disposition ist nur $3 \mathrm{mal}$ verzeichnet, Erkältung 4 mal, allein nur $1 \mathrm{mal}$. Dass letztere hier so selten in der Anamnese auftritt, ist auf die Art des Materials zurückzuführen. Im Leben von meist auf die Stube angewiesenen Personen spielt dieses Moment keine Rolle. Unter unseren Schneiderinnen findet sich thatsächlich nur eine, die sich an auch nur einmalige starke Durchnässung und Erkältung erinnerte (Fall 18). Dafür liefert wieder die Rubrik der körperlichen Anstrengung eine Ziffer, der hier ausser der Syphilis allein noch Beachtung zu schenken ist. Wir finden diese Schädlichkeit $10 \mathrm{mal}$, theils allein, theils in Verbindung mit anderen erwähnt, und zwar $8 \mathrm{mal}$ als anhaltendes Maschinenähen. Dass diese Beschäftigung verschiedentlich in ursächlichen Zusammenhang mit der Tabes gebracht worden, ist bekannt. 1882 theilte 0. Guelliot ${ }^{2}$ ) zwei solcher Beobachtungen aus Lancereaux's Klinik mit. Ein weiteres Beispiel finden wir bei Webber ${ }^{3}$ ), dann bei M. Bernhardt4).

Nach der Ansicht dieses Autors kann die dauernde Erschlitterung der Beine und des unteren Rumpfabschnittes sehr wohl zu Veränderungen im Marke Veranlassung geben. Es sei aber doch auffällig, dass trotz der grossen Verbreitung der Nähmaschine und der grossen Zahl von Individuen, die sich durch anhaltende Beschäftigung mit derselben ihren Lebensunterhalt erwerben, so relativ wenig an Tabes erkranken! Guelliot hatte die Entwicklung des Leidens bei solchen Arbeiterinnen auf die sensible Reizung, welche die fortwährende Bewegung der Beine hervorrufe („Trépidation") bezogen, während er auf die angeblich

1) Deutsche Medicin. Wochenschrift. 1897. Nr. 7.

2) L'union médicale. 1882. Nr. 2, 3, 4.

3) The Boston med. journ. 106. 1882.

4) Zur Aetiologie der Tabes. Neurol. Centralblatt. 1890. S. 710.

Deutsche Zeitschr. E. Nervenheilkunde. XII. Bd. 
dabei entstehende sexuelle Erregung keinen Werth legte. Auch Decaisn $\mathrm{e}^{1}$ ) war $z$ dem gleichen Resultate gelangt: unter 335 Maschinennäherinnen hatten sich nur 68 gefunden, die angaben, durch das Arbeiten geschlechtlich gereizt zu werden. Anders Lancereaux ${ }^{2}$ ), dessen Ansicht dahin geht, Tabes finde sich unter Weibern fast ausschliesslich bei Prostituirten oder solchen Franen, die in Folge ihrer Beschäftigung(Maschinenähen) ihre Sexualorgane der Erregung aussetzen.

Fälle von Tabes bei Maschinenarbeiterinnen finden wir dann noch in verschiedenen Statistiken, so einen bei Eulenburg ${ }^{3}$ ), drei beiHlubek, einen bei Friedrichsen, einen bei Leyden ${ }^{4}$ ).

In meinen 24 Fällen sind $19 \mathrm{mal}$ Notizen gemacht worden, ob Maschinenähen stattgefunden hatte oder nicht. Ich stelle diese Fälle der Uebersicht wegen zusammen:

L. Fälle mit Maschinenähen.

\begin{tabular}{|c|c|c|c|}
\hline Fall & Maschinenähen & Syphilis & Andere Ursachen \\
\hline $\begin{array}{r}3 \\
10\end{array}$ & $\begin{array}{l}\text { Nur im ersten Jahre der Ehe. } \\
6 \text { Jahre, dann ebensolange } \\
\text { vor Ausbruch der Tabes } \\
\text { nicht mehr. }\end{array}$ & $\begin{array}{l}\text { sicher } \\
\text { wahrscheinlich }\end{array}$ & - \\
\hline 15 & Viel. & wahrscheinlich & $\begin{array}{l}\text { Gemütbsbew egungen, An- } \\
\text { strengung durch Unter- } \\
\text { richt. }\end{array}$ \\
\hline 17 & -Viel. & wahrscheinlich & Neuropathische Belastung. \\
\hline 18 & Viel. & sicher & Einmalige Erkältung. \\
\hline 19 & $\begin{array}{l}\text { Nur ein Jahr vor Ausbruch } \\
\text { der Tabes. }\end{array}$ & wahrscheinlich & Neuropathische Belastung. \\
\hline 25 & Viel, 8 Jahre. & - & - \\
\hline 29 & $\begin{array}{l}11 \text { Jahre, aber nur } 1 \text { Jahr } \\
\text { vor Beginn der Tabes. }\end{array}$ & - & - \\
\hline 30 & $\begin{array}{l}2 \text { Jahre, nur ein Jahr vor Be- } \\
\text { ginn der Tabes. }\end{array}$ & - & - \\
\hline $\mathbf{3 3}$ & $\begin{array}{l}\text { Viel (ca. } 9 \text { Stunden täglich } \\
\text { ? Jahre lang). }\end{array}$ & verdächtig & - \\
\hline 36 & Viel (20 Jahre). & verdächtig & 一 \\
\hline 37 & $\begin{array}{l}\text { Viel (14-15 Jahre), aber eben- } \\
\text { so lange auch tabisch. }\end{array}$ & verdächtig & - \\
\hline $\mathbf{4 0}$ & Viel (9 Jahre). & verdächtig & - \\
\hline
\end{tabular}

1) La machine a coudre et la santé des ouvrières, Annales d'hygiène publ. 1870. S. 105.

2) Transactions of the international med. Congress. London 1881. U. S. 32.

3) Virchow's Archiv. 1885.

4) Arbeiten aus der I. med. Klinik. 1888/89. 
Also unter 13 Fällen 7, höchstens 8, wenn man Nr. 10 noch mit hinzurechnen will, in denen bis zum Beginne der Tabes angestrengtes Maschinenähen stattgefunden hatte. Unter diesen befinden sich 1 mit sicherer, 3 mit wahrscheinlicher Syphilis, 3 verdächtige.

Wir verfügen nun noch über weitere 6 Kranke, die sich niemals mit Maschinenähen beschäftigt hatten, bei denen aber die Syphilis eine ganz erhebliche Rolle spielt:

II. Fälle ohne Maschinenähen.

\begin{tabular}{|c|c|c|}
\hline Fall & Syphilis & Andere Ursachen \\
\hline $\begin{array}{c}7 \\
12 \\
16\end{array}$ & $\begin{array}{l}\text { sicher } \\
\text { sicher } \\
\text { verdächtig } \\
\text { verdächtig } \\
\text { sicher } \\
\text { sicher }\end{array}$ & $\begin{array}{l}\text { neuropathische Belastung. } \\
\text { anstrengende } \\
\text { arbeit. } \\
\text { neuropathische Belastung. }\end{array}$ \\
\hline
\end{tabular}

Also zwei Drittel mit sicherer Syphilis, ein Drittel mit Verdacht darauf. Die übrigen Schädlichkeiten sind von untergeordneter Bedeutung.

Wir können diese kleine Tabelle noch weiter ausdehnen und anch diejenigen Fälle hinzurechnen, in denen so wenig Maschinenarbeit geleistet worden ist, dass sie für die Entstehung der Tabes nicht verantwortlich gemacht werden kann. Es sind dies (s. die erste Tabelle) 5, darunter 1 mit sicherer, 1 mit wahrscheinlicher Syphilis, 1 verdächtig. Dann hätten wir also unter 11 Fällen 5 mit sicherer, 1 mit wahrscheinlicher Syphilis und 3 verdächtige. Diesen stehen 8 mit anhaltendem Maschinenähen gegenüber, unter denen sich aber nur einer [Fall 25] befindet, bei dem für Syphilis keine Anhaltspunkte vorlagen. Die Schädlichkeit, die in 6 Fällen, in denen die Maschine nie benutzt worden ist, maassgebend erscheint, kehrt in fast allen anderen wieder. Es ist demnach gewiss gerechtfertigt, bei der Mehrzahl in der Syphilis die eigentliche Grundlage der Tabes zu sehen.

Um den Ueberblick über das Verbältniss der Näbmaschinenarbeit zur Aetiologie der Tabes zu erweitern, habe ich eine grössere Anzahl nicht tabischer nervenkranker Näherinnen zum Vergleiche herangezogen. Ich wählte hierbei solche zwischen dem 30. und 50. Lebensjahre, einige wenige darüber, aber nicht ubber 55 Jahre aus, um in den Grenzen desjenigen Lebensalters zu bleiben, in denen sich die Tabes entwickelt. 
Mit Recht fordert Hermanides ${ }^{1}$ ) ein solches Vergleichsverfahren, das übrigens auch schon von $\mathrm{Erb}^{2}$ ) in seiner bekannten Gegenstatistik angewandt worden ist. Es sind 74 Fälle (vorzugsweise Neurasthenie, dann Hysterie, Neuralgien etc.). 4 darunter waren sicher syphilitisch, 1 verdächtig, zusammen 6,8 Proc. $46=62$ Proc. haben angestrengt, mindestens 5 Jahre lang, vielfach weit länger, bis zu 22 Jahren Maschine genäht. Es handelt sich hier stets um die Fussmaschine. Handmaschinen werden von Berufsarbeiterinnen so gut wie nie benutzt.

Weiter verfüge ich durch die Güte einiger Collegen tuber 60 Fälle von nicht nervenkranken Patientinnen derselben Krankenkasse, ebenfalls in dem genannten Alter. Von diesen haben $39=65$ Proc. mindestens 5 Jahre hindurch schwere Maschinenarbeit verrichtet. Zusammen mit den ersteren sind es also 134 Fälle, darunter 85 Maschinennäherinnen $=63$ Proc. Diese Zahl gestattet wohl die Annahme, dass die Tabes unter Schneiderinnen sehr viel häufiger sein müsste, wenn der Nähmaschine eine directe Einwirkung auf die Entstehung dieses Leidens zugeschrieben werden könnte. Man beachte hierzu, dass die Ortskrankenkasse der Schneider ca. 24000 weibliche Mitglieder besitzt, von denen gewiss die Hälfte, unseren Erhebungen nach wahrscheinlich weit mehr, sich in intensiver Weise mit dem Treten der Nähmaschine beschäftigt. Wir dürfen danach wohl den Schluss, den wir zunächst nur auf nnsere Fälle angewandt haben, verallgemeinern und der Nähmaschinenarbeit an sich überhaupt den Platz unter den Tabesursachen streitig machen. Damit fäll aber wieder ein Factor, der für die Aetiologie dieses Leides in Anspruch genommen worden ist. Um so enger ziehen sich die Kreise um dasjenige Moment zusammen, das den meisten Fällen seinen Stempel aufdrückt, die Syphilis. Redlich hat die Ansicht ausgesprochen, dass für eine kleine Zahl von Fällen andere infectiös-toxische Processe als der Syphilis gleichwerthig, wie auch Erkältungen und Trauma in Betracht kommen, fernẹr, dass für die Entwicklung der Tabes, besonders für deren progressiven Charakter, noch weitere Hülfsmomente, in erster Linie Ueberanstrengungen, speciell der uateren Extremitäten und Erkältungen mitzusprechen haben. Unser Material liefert für alles dies keine Stützpunkte. - Man hat auch verschiedentlich die Quecksilberbehandlung der Syphilis mit argwöhnischen Augen betrachtet, was aber ebenso oft, erst jüngst wieder von $\mathrm{Erb}^{3}$ ), zurn̈ckgewiesen worden ist. Es sei hier an Four-

1) Virchow's Archiv 148. S. 521.

2) Die Aetiologie der Tabes. Volkmann's klinische Vorträge. Nr. 53.

3) Deutsche Zeitschrift f. Nervenheilkunde. Bd. 11. 1897. S. 261. 
nier's bekannte Statistik ${ }^{1}$ ) erinnert, nach der die Zahl der späteren Tabiker um so mehr abnimint, je energischer und länger die Quecksilberbehandlung gewesen ist. So stehen z. B. 108 Tabikern, die eine solche nur 3-7 Monate gebraucht haben, 12, die $2 \mathrm{Jahre,} \mathrm{6,} \mathrm{die} \mathrm{3,}$ nur 2, die 4 Jahre darunter gehalten worden sind, gegenüber.

Die Achillesferse dieser Aufstellung ist dem grossen Kliniker freilich nicht entgangen; man könnte nämlich entgegnen, die Zahl der nur kurze Zeit behandelten Syphilitiker sei die grössere, deshalb müssen sie auch unter den späteren Tabikern mit der grösseren Ziffer vertreten sein. Fournier lässt diesen Einwurf allerdings nicht zu. Derselbe wird auch durch die sich neuerdings geltend machende Ansicht in den Hintergrund gedrängt, dass die Tabes gar nicht mit Vorliebe der mangelhaft behandelten Syphilis folgt.

Nach Collins ${ }^{2}$ ) scheint weder gründliche Behandlung der ursprünglichen Lues die Entwicklung der Tabes zu verhindern (was ja auch schon Fournier und Erb beobachtet haben), noch ungenügende sie zu befördern. Redlich schliesst sich dieser Annahme an.

Einen weiteren Einblick in diese Verhältnisse suchte ich dadurch zu gewinnen, dass ich die Intervalle zwischen Syphilis und Tabes trennte, je nachdem Quecksilber gebraucht worden ist oder nicht.

Unter meinen 23 weiblichen Tabikern mit sicherer oder fast sicherer vorangegangener Syphilis liess sich $21 \mathrm{mal}$ das Intervall mit mehr oder weniger Genauigkeit bestimmen.

\section{Fälle mit Quecksilberbehandlung.}

\begin{tabular}{|c|c|c|}
\hline Fall & Curen & $\begin{array}{l}\text { Intervall } \\
\text { in Jahren. }\end{array}$ \\
\hline $\begin{array}{r}4 \\
8 \\
14\end{array}$ & $\begin{array}{c}\text { Injectionseur im Beginne der Infection } \\
" \quad \text { danach Pillen } \\
" \quad \text { später kurz Jodkalium und Einreibungen }\end{array}$ & $\begin{array}{r}9 \\
10 \\
4\end{array}$ \\
\hline 21 & $\begin{array}{l}3 \text { Wochen Schmiercur beim Exanthem, danach } 1 / 4 \text { Jahr } \\
\text { Jodkalium }\end{array}$ & $\begin{array}{l}\text { bald nach } \\
\text { dem Exan- } \\
\text { them. }\end{array}$ \\
\hline 25 & $\begin{array}{l}\text { Injectionscur im Beginne der Lues, Schmiercur beim } \\
\text { Recidiv }\end{array}$ & 5 \\
\hline 31 & Injectionscur bei Roseola & 3 \\
\hline
\end{tabular}

1) Enquête sur la prétendue action tabétogène du traitement mercurial. Gazette hebdom. 1891. S. 606.

2) The influence of anti-syphilitic treatment etc. The Post Graduate. XI. S. 287. Referirt im Neurol. Centralblatt. 1897. S. 276 (das Original war mir leider nicht zugänglich). 


\section{Fälle ohne Quecksilberbehandlang.}

\begin{tabular}{|c|c|c|c|c|c|c|c|c|c|c|c|c|c|}
\hline Fall & 3 & 11 & 10 & $12^{*}$ & $15^{*}$ & 17 & $18^{*}$ & 23 & 24 & 27 & 28 & $39 *$ & $41^{*}$ \\
\hline $\begin{array}{c}\text { Intervall- } \\
\text { jahre. }\end{array}$ & 20 & 13 & 2 & 6 & 11 & 3 & 3 & 18 & 17 & \begin{tabular}{|l|}
$\mathbf{s e h r}$ \\
$\mathbf{k} u r z$
\end{tabular} \mid & 9 & 3 & 11 \\
\hline
\end{tabular}

(Die mit * bezeichneten Fälle haben erst nach sicherem Ausbruch der Tabes kurze Inunctions- oder Jodkaliumscuren gebraucht.)

Zwei Fälle (Nr. 7 and 34) sind im Beginn der Infection mit Jodkalium behandelt worden.

Die Intervalle betrugen hier 6 und 4 Jahre. Die Intervalle sind danach bei den nicht behandelten Fällen im Allgemeinen länger; in 6 von 13 betragen sie 10-20 Jahre, während sie sich in den behandelten kein Mal über 10 Jabre erheben.

Die geringe Zahl der Fälle veranlasste mich, auch mein Material an männlichen Tabikern auf diese Frage hin zu prüfen. Ich verfüge hier uber 37 mit Voranfgang von sicherer Syphilis oder als hart bezeichnetem Schanker.

I. Fälle mit äblicher, zum Theil energischer Quecksilberbehandlung i. Sa. 22.

Intervalle: 2,5 (3 mal), 6 (4 mal), 7 (2 mal), $8,9(3 \mathrm{mal}), 10,11,12,13,17,18(2 \mathrm{mal})$, 22 Jahre.

Im Mittel 9,6 Jahre.

II. Fälle ohne Quecksilberbehandlung i. Sa. 15.

Intervalle: 2,7 (3 mal), 10, 11, 12 (2 mal), 13, 20 (3 mal), 21, 23 Jahre.

Im Mittel 13 Jabre.

Also auch hier die meisten grösseren Intervalle bei den nicht behandelten Fällen. Stellen wir die grössten (20 Jahre und darüber) zusammen, so finden wir sie in

$$
\begin{aligned}
& \text { Tabelle I } 1 \mathrm{mal} \text {, } \\
& \text { " II } 5 \mathrm{mal} \text {. }
\end{aligned}
$$

Umgekehrt zeigen sich die kleinen Intervalle (bis zu 10 Jahren) in Tabelle I $13 \mathrm{mal}$,

$$
\text { III } 5 \mathrm{mal} \text {. }
$$

Wir sehen somit die grössere Zahl der längsten Intervalle bei den nicht behandelten, die der kürzesten bei den mit Quecksilber behandelten Tabikern. Nehmen wir einer Berechnung Friedrichsen's ${ }^{1}$ ) gemäss die mittlere Grösse des Intervalls mit 9 Jahren an, so erhebt

1) 1. c. 
sich die Mehrzahl der nicht behandelten Fälle (zum Theil weit) über dieses Maass, während sich die Mehrzahl der behandelten darunter hält. - Es wäre nun möglich, dass in den Fällen mit den kürzeren Intervallen zufällig gewisse, den Ausbruch der Tabes beschleunigende Hiblfsursachen vorhanden gewesen sind. Solche haben aber bei den hier in Betracht kommenden 15 Kranken mit Quecksilberbehandlung $7 \mathrm{mal}$ gar nicht vorgelegen, in den 4 Fällen mit einem Intervall bis zu 5 Jahren 3 mal nicht.

Bei den Kranken, denen kein Quecksilber einverleibt worden ist, sind überhaupt nur 4 mal Schädlichkeiten ausser der Syphilis angegeben. Davon fallen 3 auf Fälle mit Intervallen von 10-20 Jahren, und nur einer auf ein solches von 7 Jahren. Von dieser Seite kann der Unterschied also nicht herrühren. Nun könnte erwartet werden, dass die besonders lange und energisch behandelten Syphilitiker auch besonders früh zur Tabes gelangt seien. Thatsächlich finden sich unter unseren 12 Fällen dieser Art 10 mit den kürzeren Intervallen, 2 mit 5, 4 mit 6 , je 1 mit 8 und 9,2 mit 10 Jahren.

Selbstrerständlich wäre es roreilig, aus diesen doch nur kleinen Zahlen verallgemeinernde Schlüsse ziehen zu wollen, das muss einem beweiskräftigeren Materiale überlassen bleiben. Es darf auch nicht äbersehen werden, dass das Intervall kaum je scharf abzugrenzen, dass mit Idiosyncrasie und Disposition zu rechnen ist, die Zahlenunterschiede sind aber doch $\mathrm{zu}$ auffallend, um nicht dem Gedanken Raum zu geben, dass die Tabes bei unseren Kranken der normal, bez. energisch behandelten Syphilis im Allgemeinen schneller gefolgt ist, als der nicht behandelten. Jedenfalls würde das Resultat die Ansicht Collin's stützen. - Wenn das Quecksilber überhaupt eine Rolle bei der Entwicklung der Tabes spielt, so kann dies allerdings nur so zu verstehen sein, dass es als antiplastisches, im Sinne Edinger's ${ }^{1)}$ ersatzstörendes Agens der specifischen oder postsyphilitischen Toxinwirkung die Wege ebnet. Ein vorsichtiges Regimen wird diese Schädlichkeit gewiss auf ein Minimum reduciren. So lassen sich die günstigen Erfolge, die Erb u. A. mit der Quecksilberbehandlung bei Tabes erzielt haben, sehr wohl mit der genannten Anschauung in Einklang bringen. - Es gehört aber wohl ausser der Syphilis und den depotenzirenden Momenten noch ein drittes dazu, um die Entstehung der Tabes herbeizuführen, das ist die erst kürzlich wieder ron Benedict ${ }^{2}$ ) betonte Disposition, die in den betreffenden sensiblen Neu-

1) Eine neve Theorie über die Ursachen einiger Nervenkrankheiten. Volkmann's klinische Vorträge. 1894. Nr. 6.

2) Verhandlungen des internat. med. Congr. zu Moskau. Neurol. Centralblatt. 1897. Nr. 18. S. 875 . 
ronen einen Locus minoris resistentiae schafft. Sonst würde die Syphilis, deren Ausbreitung von Erb ${ }^{1}$ ) auf 12,1 Proc., von A. Blaschko ${ }^{2}$ ) auf 10-12 Proc., also nahezu gleich geschätzt wird, häufiger Tabes im Gefolge haben.

Bis hierher haben wir uns bei unserem Versuche, einen, wenn auch nur bescheidenen, Beitrag zur Lehre von dem syphilitischen Ursprung der Tabes zu liefern, lediglich der Statistik bedienen müssen. Wenden wir uns jetzt zur Einzelbetrachtung unserer Beobachtungen, so fallen einige ins Auge, die beredter für den Zusammenhang der beiden Leiden sprechen, als Zahlen. Es sind dies zanächst die Fälle von Tabes oder Tabes und Paralyse bei Eheparen.

Wir besitzen in der Literatur eine ganze Reibe solcher Mittheilungen, so von v. Strümpell ${ }^{3}$ ) (Mann und Frau typische Tabes, beide luetisch), von Goldflam ${ }^{4}$ ) (ebenso), dann von $\operatorname{Erb}^{5}$ ), ganz besonders ron Mendel \%), der 18 Fälle von Tabes, Paralyse, oder Tabes und Paralyse eigener Beobachtung zusammengestellt hat, unter denen $8 \mathrm{mal}$ beide Ehegatten, $5 \mathrm{mal}$ nur der Ehemann syphilitisch gewesen waren.

Meine Krankengeschichten weisen 2 mal Tabes bei Ehegatten auf: Fall 31 und 39. Im ersten Falle hatte der Mann zugegeben, syphilitisch gewesen zu sein. Derselbe zeigte reflectorische Starre der erheblich nngleichen Pupillen, einseitige Abschwächung des Kniephänomens, ausgebreitete Parästhesien. Typische Tabes war auch bei dem Manne der zweiten Patientin zu constatiren (reflectorische Pupillenstarre, W estphalsches Zeichen, Blasenparese, Impotenz, Hypalgesie); er gab nur einen Tripper anfangs der $20 \mathrm{er}$ Jahre zu (hatte aber später mehrfach Iritis), inficirte damals seine Braut. Bei zwei weiteren Fällen (15 und 23) wurde angegeben, dass der Ehemann an Dementia paralytica gestorben sei. In Fall 15 ist derselbe syphilitisch gewesen („Beulen" auf dem Kopf, Iritis). Die Ehe wurde wegen Ansteckung der Patientin geschieden. Fall 23 giebt über Syphilis des Mannes keine Auskunft, doch kann man aus den Geburten der Frau Verdacht schöpfen. Die Infection dírfte eventuell nach dem ersten Kinde erfolgt sein.

Einen weiteren Anhaltepunkt für den syphilitischen Ursprung der Tabes finden wir in den Fällen mit später Infection und später Tabes. Wir. haben oben 2 dieser Art kennen gelernt. In Fall 14 bekam die

1) Die Aetiologie der Tabes.

2) Syphilis und Prostitation. Berlin 1893.

3) Neurologisches Centralblatt 1888. 8. 122.

4) Deutsche Zeitechrift für Nervenheilkunde. 1892.

5) Die Therapie der Tabes.

6) Neurologisches Centralblatt. 1895. S. 335. 
48jährige bis dahin gesunde Frau, Mutter von 8 Kindern, durch den Kuss eines Pensionärs ein Ulcus durum auf der Lippe, im 52. Jahre begann die Tabes. Der andere Fall (26) betrifft eine Hebamme, die sich im 45. Jahre am Finger inficirte und mit 50 Jahren an Tabes erkrankte. [Beispiele von vorausgegangener specifischer Fingerinfection bei Tabikern finden wir z.B. bei Althaus ') (Arzt), bei Eulenburg ${ }^{2}$ ) und HIubek ${ }^{3}$ ) (Hebammen).] Andere Anbaltspunkte fehlen in beiden Fällen.

Für noch bei der Tabes bestehende Syphilis habe ich nur einige Beläge: Fall 18 und 21, dazu noch Fall 40 mit gewissen Verdachtsmomenten.

Was die einzelnen Symptome unserer Fälle anbetrifft, so stehen unter den Initialerscheinungen die lanzinirenden Schmerzen wie gewöhnlich im Vordergrunde, $22 \mathrm{mal}$ unter 37 Fällen, in denen die Anamnese über den Beginn Auskunft gab, $=$ ca. 60 Proc. (Leimbach ${ }^{4}$ ) hatte unter Erb's Material 88,25 Proc. gefunden.) Typische Migräne ist nur einmal als Anfangs-, einmal als späteres Symptom verzeichnet. Nach Berger ${ }^{5}$ ) und Oppenheim ${ }^{6}$ ) (letzterer hat unter 85 Tabeskranken 12 mit Migräne, darunter 10 Frauen, beobachtet), wären mehr Fälle zu erwarten gewesen. $3 \mathrm{mal}$ hat Schwäche eines Beins, ebenso oft Augenmuskellähmung, $1 \mathrm{mal} \mathrm{Gürtelschmerz} \mathrm{die} \mathrm{Scene} \mathrm{eröffuet,} 2 \mathrm{mal}$ wurden Parästhesien, $1 \mathrm{mal}$ gastrische Krisen, $4 \mathrm{mal}$ cephalische Symptome (Bewusstlosigkeit, Schwindel, Kopfschmerz) angegeben.

Besondere Aufmerksamkeit wurde dem Ulnaris- und Peroneussymptom geschenkt.

Seitdem Biernacki i ) die Unempfindlichkeit des Nervus ulnaris auf Druck in den Sulcus ulnaris als ein Attribut der Hinterstrangsklerose gedeutet, ist diese Erscheinung verschiedentlich aufgesucht worden. Orschansk $y^{8}$ ), der seine Untersuchungen an einem grossen Materiale, vornehmlich der Mendel'schen Poliklinik angestellt hat, ist zu der Ueberzeugung gekommen, das Ulnarissymptom sei nichts Charakteristisches für Tabes. Dem gegenüber ist es Sarbó ó ${ }^{9}$ ) wieder gelungen, unter 15 Tabikern 11 mit doppelseitiger Ulnarisanalgesie und ebensoviele mit doppelseitiger oder einseitiger Peroneusanalgesie zu finden.

1) The British medical journal. 1884. Nr. 31.

2) a. a. 0 . S. 18.

3) a. a. 0 .

4) Deutsche Zeitschrift für Nervenheilkunde. XX. 1889. S. 131.

5) Berliner ärztliche Zeitschrift. 1884. Nr. 13.

6) Berliner klinische Wochenschrift 1884. S. 603.

7) Neurol. Centralblatt. 1894. S. 242.

8) Ueber die Ulnarisanalgesie als Tabessymptom. Berliner Dissertation. 1895.

9) Neurol. Centralblatt. 1896. S. 351. 
Unter den äbrigen Fällen war die Empfindlichkeit der betreffenden Nerven nur in einem normal, sonst wenigstens herabgesetzt.

Von unseren Fallen ist in 22 auf das Ulnarissymptom, in 12 gleichzeitig auf das Peroneussymptom geachtet worden: das erstere war $14 \mathrm{mal}$, das letztere $12 \mathrm{mal}$ nachzuweisen. Diese Zahlen fallen um so mehr ins Gewicht, als sowohl Hess ${ }^{1}$ ) wie Sarb ó ${ }^{2}$ ) darauf aufmerksam gemacht haben, dass das Ulnarissymptom bei Frauen seltener ist, was Sarbó auf die grössere Empfindlichkeit derselben bezieht.

Die Gegenprobe wurde an 57 Patientinnen mit verschiedenen functionellen Störungen angestellt. Hier war das Ulnarissymptom nur 4 mal anzutreffen. Danach dürfte demselben eine gewisse Bedeutung dosh nicht abzusprechen sein. -

Eine eigenartige Erscheinung zeigt Fall 39. Diese Kranke mit doppelseitiger totaler Opticusatrophie hat seit 2 Jahren einen Tag einen dunklen, den anderen einen hellen (grauen) Schein vor den Augen.

Der Wechsel dieser Tage ist ein ganz regelmässiger. Der "graue Tag" beginnt gegen 4 Uhr Morgens und hält fast genau seine 24 Stunden an, um dann wieder dem "schwarzen Tage" Platz zu machen. Ich bin diesem Symptom bisher nur einmal begegnet. Der Fall betrifft einen 56jährigen Collegen mit Amaurose in Folge von (nicht tabischer) retrobulbärer Neuritis. Auch hier vollzieht sich der Wéchsel des "hellen" und "dunklen" Tages seit Jahren mit grösster Gleichmässigkeito Eine heftige Gemüthsbewegung hatte den Anstoss dazu gegeben. In dem erstgenannten Falle konnte kein Grund angeführt werden. Diese Patientin befindet sich an dem grauen Tage wohler, der andere Kranke leidet dagegen schwer unter dem hellen, an dem er gleichsam in blendenden Schnee sieht. Wir haben es hier wohl mit einem functionellen Reizzustande zu thun. Die fast auf die $\mathrm{Uhr}$ eingestellte Regelmässigkeit des Intermittirens erinnert an gewisse hysterische Zustände.

Einige seltenere Complicationen mögen hier noch Beachtung finden:

1. mit Basedow'scher Krankheit (Fall 17). Joffro ${ }^{3}$ ) rechnet nur die ausgebildeten Formen zu den Complicationen, während er die unvollkommenen als Tabessymptome deutet. In unserem Falle ist ein ausgeprägter Exophthalmus allerdings nicht vorhanden, doch lassen ihn die erhebliche Struma, die andauernde Pulsfrequenz von 120, die Schweisse, die Abmagerung, die Herabsetzung des Leitungswiderstandes

1) Neurol. Centralblatt. 1896. Nr. 1. S. 40.

2) a. a. 0 .

3) Des rapports de l'ataxie locomotrice progr. et dn goitre exophthalmique. Gazette hebdom. 1889. 
der Haut den klinischen Beiträgen von Möbius ${ }^{1}$ ), Timotheef ${ }^{2}$ ) u. A. wohl an die Seite stellen. Ob der in einem solchen Falle von P. Marie and G. Marinesco ${ }^{3}$ ) erbobene Befund (symmetrische Degeneration des solitären Bündels und der aufsteigenden Trigeminuswurzel) der Basedow'schen Krankheit zukommt, wie diese Autoren annehmen, oder ob er eine Manifestation der Tabes ist, dürfte erst durch weitere Beobachtungen entschieden werden. -

2. mit Diabetes mellitus (Fall 13). Hier kann bekanntlich ein diagnostischer Irrthum dadurch hervorgerufen werden, dass sich im Verlaufe eines Diabetes Neuritiden entwickeln, die einen tabesähnlichen Symptomencomplex vortäuschen. Im vorliegenden Falle hatte sich aber der jetzt seit Jahren bestehende Zuckergehalt (anhaltend bis 4,3 Proc.) erst gezeigt, nachdem schon längst Pupillenstarre, Opticusatrophie etc. festgestellt war. In 0ppenheim's Fall ${ }^{4}$ ) fand sich nur 0,7-1, 3 Proc., in Reumont's ${ }^{5}$ ) auch nur bis 1 Proc. Zucker.

3. mit Bulbärparalyse (Fall 1). Die Beobachtung dieser seltenen ${ }^{6}$ ) Complication konnte leider nur kurze Zeit bindurch stattfinden.

4. mit traumatischer Hysterie (Fall 9).

Wir können unseren Gegenstand nicht verlassen, ohne ein Argument zu berühren, das verschiedentlich (Eulenburg, Edinger, Hermanides u. A.) gegen den Zusammenhang von Tabes und Syphilis in die Wagschale geworfen worden ist: die Seltenheit der Tabes bei den Prostituirten, die doch so ungemein häufig von Syphilis befallen werden. Eding er ${ }^{7}$ ) erklärt dies im Sinne der Functionstheorie mit der mehr sitzenden Lebensweise dieser Individuen, Redlich ${ }^{8}$ ) ist der Ansicht, es liege zum Theil daran, „dass in dem Alter, in dem die Frauen an Tabes erkranken, die Zeiten der Prostitution längst vorüber sind und in der Anamnese letztere möglichst versteckt wird". - Vielleicht hat man auch die Krankheit hier nur so häufig vermisst, weil man ausgebildete Formen erwartete, die sich bei dem langsamen Verlaufe der Tabes allerdings erst in späteren Jahren zeigen können.

Bei dieser Sachlage erschien es mir nothwendig, die Tabes unter den Prostituirten direct aufzusuchen. Ein dazu geeignetes Material

1) Deutsche Zeitschrift für Nervenheilkunde. Bd. I. S. 400 .

2) Berliner Dissertation. 1893,

3) Revue neurologique. 1893. S. 10.

4) Berliner klinische Wochenschrift. 1885. S. 815 .

万) Berliner klinische Wochenschrift. 1886. S. 207.

6) Charcot, La syndrome paralysie labio-glosso-laryngée progr. dans le tabes. Le Progrès médical. 1893.

7) 1. c. S. 106 .

8) 1. c. S. 129 . 
bot mir die Station für geschlechtskranke Weiber im hiesigen städtischen Obdach, die sich nur aus unter sittenpolizeilicher Controle stehenden, also gewissermaassen legitimirten Puellis publicis recrutirt. ${ }^{1}$ )

Es kamen zur Untersuchung 184 Fälle. Daron standen im Alter von

\begin{tabular}{|c|c|c|}
\hline \multicolumn{3}{|c|}{ 14 Jahren: 2} \\
\hline $15-19$ & $"$ & 79 \\
\hline $20-24$ & & 67 \\
\hline $25-29$ & " & 16 \\
\hline $30-34$ & $"$ & 13 \\
\hline
\end{tabular}

$\begin{array}{rrr}35-39 & \text { Jahren: } & 1 \\ 40-44 & " & 2 \\ 45-49 & " & 2 \\ 55 & " & 1 \\ 60 & " & 1\end{array}$

Wir entnehmen daraus zunächst, dass die Zahl der Prostituirten jenseits der 30 er Jahre, ja schon vom 25. an erheblich abnimmt. Dies könnte hier allerdings seinen Grund darin haben, dass die meisten in diesem Alter für Syphilis schon immun sind und so zur Einlieferung in das Krankenhaus seltener Veranlassung geben. Ich verfüge aber noch tber eine andere Zahlenreibe, die mir Herr Bezirksphysikus Dr. Granier freundlichst überlassen hat: von 221 Prostituirten, die zur gewöhnlichen ärztlichen Untersuchung erschienen, standen im Alter von

$\begin{array}{lll}\text { 17-24 Jahren: } & 77 \\ 25-29 & 79 \\ 30-34 & 7 & 26 \\ 35-39 \quad " & 25\end{array}$

$\begin{array}{crr}40-44 & \text { Jahren } & 10 \\ 48 & & 3 \\ 52 & " & 1\end{array}$

Also anch hier rapide Abnahme jenseits der 30 er Jahre.

Unter den von mir untersuchten Prostituirten fanden sich nun 5 mit tabischen Symptomen:

1. 27 Jahre: Pupillen reflectorisch starr, Blasenparese, Analgesie an den unteren Extremitäten, lanzinirende Schmerzen daselbst. Vor 11 Jahren Injectionscur in der Charité.

2. 28 Jahre: Pupillen ungleich, reflectorisch starr, Kniephänomen einseitig sehr schwach. Ausgebreitete Analgesie an den unteren Extremitäten und am Rumpfe. Vor 13/4 Jahren Schmiercur.

3. 47 Jahre: Pupillen reflectorisch starr, lanzinireude Schmerzen in den Beinen.

4. 47 Jahre: Pupillen reflect. starr, Romberg's und Westphal's Zeichen, Blasenparese. - Vor 20 Jahren geschlechtskrank, nur örtlich behandelt.

1) Ich sage Herrn Prof. G. Behrend und Herrn Collegen E. Levin für ihr gefalliges Entgegenkommen auch an dieser Stelle meinen wärmsten Dank. 
5. 55 Jahre: Pupillen ziemlich eng, reflect. starr. Angeblich keine Syphilis.

Da wir mit einem gewissen Intervall, im Durchschnitt (s. o.) 9 Jahre, zu rechnen haben, so brauchen wir auch hier, trotz der gewiss oft frühen Infection dieser Individuen, den Beginn des Alters, in dem Tabes aufzutreten pflegt, nicht vor dem 25. Jahre anzunehmen. Dann scheiden aber 148 aus und haben nur 36 die oben genannten 5 Fälle gestellt, d. i. 14 Proc. Tabes bei Prostituirten im tabesfähigen Alter.

Diese gar nicht kleine Zahl bildet aber doch nur die unterste Grenze, da später noch mehr der Tabes verfallen können. Der Procentsatz ist auch dann nicht gering, wenn wir alle 36 Prostituirte als syphilitisch inficirt betrachten, obwohl 10 davon nie eine Cur gebraucht haben wollen. ${ }^{1}$ )

Sollten weitere Untersuchungen meine Erfahrungen bestätigen, so wird man auch diesen Einwand gegen den syphilitischen Ursprung der Tabes nicht wohl aufrecht halten können.

1) Raff (Archiv f. Dermatologie. Band 36, S. 16), hält Lesser's Ausspruch (Lehrbuch der Geschlechtskrankheiten. 1888. S. 302), jede Prostituirte werde syphilitisch inficirt, nicht für zutreffend. Auch Sederholm und Wwedensky (beide citirt bei Raff) haben 35,6 bezw. 39,6 Proc. frei von Syphilis gefunden. 